\title{
Factores asociados al consumo de sustancias psicoactivas en población atendida en un hospital de alta complejidad de Tunja: Estudio de casos y controles.
}

\section{Factors associated with the consumption of psychoactive substances in a population attended in a hospital in Tunja: Case-control study.}

\author{
Juan Carlos Alba Maldonado ${ }^{1}$, Ledmar Jovanny Vargas $\mathbf{R} /$ guez $^{2}{ }^{-}$, Paola Andrea Bolívar ${ }^{3}$, Sergio $^{-}$ \\ Andrés Granados López ${ }^{3}$, Marly Alexandra Fonseca Leal ${ }^{4} \odot$, Geraldine Vanesa Ayala Cuervo ${ }^{5}$, Andrés \\ Felipe Barajas Santos ${ }^{6}$
}

\author{
Hospital Universitario San Rafael, Tunja. \\ ${ }^{2}$ Hospital Universitario San Rafael, Universidad de Boyacá, Tunja. \\ ${ }^{3}$ Universidad de Boyacá, Tunja. \\ ${ }^{4}$ Universidad de Boyacá, Tunja. Centro de salud San Vicente Ferrer Saboya. \\ ${ }^{5}$ Universidad de Boyacá, Tunja. Ese centro salud Combita \\ ${ }^{6}$ Universidad de Boyacá, Tunja. Ese san Cayetano de guapota
}

*Dirigir correspondencia a: lejovaro@gmail.com

\begin{tabular}{l}
\hline Proceso Editorial \\
\hline Recibido: 021120 \\
Aceptado: 160321 \\
Publicado: 111121 \\
\hline
\end{tabular}

DOI 10.17081/innosa.139

(c) Copyright 2021

Bolívar $\mathrm{P}^{1}$ et al.

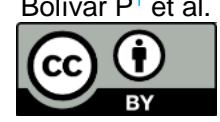

\section{RESUMEN}

Introducción: El uso y abuso de sustancias psicoactivas (SPA) es un problema de salud pública, que trae consigo consecuencias negativas a nivel económico, laboral, familiar, social y académico en los individuos que consumen. Objetivo: Determinar los factores asociados al uso de sustancias psicoactivas en pacientes atendidos por el servicio de psiquiatría en el Hospital San Rafael durante el año 2018. Metodología: Estudio de casos y controles. Se revisaron un total de 309 historias clínicas, divididas en 103 casos y 206 controles. Resultados: La pelea entre padres, consumo de sustancias por parte de un familiar, vivir en zona urbana, estar desempleado, núcleo familiar disfuncional son factores de riesgo, mientras que ser del sexo femenino y tener hijos son factores que disminuyen la probabilidad de consumir sustancias psicoactivas. Conclusiones: Los factores de riesgo para el consumo de sustancias psicoactivas son los conflictos familiares, antecedente de consumo de sustancias por parte de un familiar, vivir en zona urbana y núcleo familiar disfuncional; mientras que el sexo femenino y tener hijos son factores que disminuyen la probabilidad de consumir sustancias psicoactivas. La validez interna es adecuada para la población que sea atendida por el servicio de psiquiatría del Hospital San Rafael de Tunja, mientras que para la validez externa y la generalización a otros servicios se debe tener en cuenta que cumpla con los criterios analizados en el estudio.

Palabras clave: Sustancia psicoactiva; Uso de sustancias; Abuso de sustancias; Servicios de salud mental; relación familiar; desempleado

\begin{abstract}
Introduction: The use and abuse of psychoactive substances (PAS) is a public health problem, which brings negative consequences at the economic, work, family, social and academic levels in the individuals who consume. Objective: To determine the factors associated with the use of psychoactive substances in patients treated by the psychiatry service at Hospital San Rafael during 2018. Methodology: Case-control study. A total of 309 medical records were reviewed, divided into 103 cases and 206 controls. Results: The fight between parents, substance use by a family member, living in an urban area, being unemployed, a dysfunctional family nucleus are risk factors, while being female and having children are factors that decrease the probability of using substances psychoactive. Conclusions: The risk factors for the consumption of psychoactive substances are family conflicts, a history of substance use by a relative, living in an urban area and a dysfunctional family nucleus; while the female sex and having children are factors that decrease the probability of consuming psychoactive substances. Internal validity is adequate for the population that is cared for by the psychiatry service of Hospital San Rafael de Tunja, while for external validity and generalization to other services it must be taken into account that it meets the criteria analyzed in the study. Keywords: Psychoactive substance; Substance use; Substance abuse; Mental health services; family relationship; unemployed
\end{abstract}




\section{INTRODUCCIÓN}

El término sustancia psicoactiva corresponde a cualquier sustancia natural o sintética que altere de alguna forma el funcionamiento del sistema nervioso central (SNC) y, en concreto, la producción de neurotransmisores, ${ }^{(1,2)}$ El término consumo, según Toprak et al, es la conducta de entrar en contacto con la droga, ya sea por vía oral (tragada o masticada), inyectada, fumada o por absorción anal o vaginal; de igual manera, policonsumo simultáneo es la coingestión de diferentes sustancias al mismo tiempo(1). La marihuana, el alcohol, la cocaína, el tabaco (cigarrillo) y basuco son ejemplos de este tipo de sustancias (2).

Villalobos et al. (2011) explican que los efectos de las sustancias psicoactivas son diversos y dependen del tipo de sustancia, como también de la cantidad o frecuencia de consumo. Eso sí, todas pueden provocar sensaciones placenteras de euforia o desesperación y hacen que el consumidor incremente sus niveles de utilización para eliminar efectos desagradables que provienen del de la interrupción a ellas (ํ).

El consumo de sustancias psicoactivas (SPA) en el mundo es un problema de salud pública $(2,4,5)$, que tiene un impacto en la salud, la economía, el rendimiento académico y la convivencia familiar y social del individuo consumidor (). Según el Informe Mundial de Drogas 2017 de Oficina de las Naciones Unidas contra la Droga y el Delito (UNODC), un cuarto de billón de personas, o cerca del $5 \%$ de la población adulta, entre los 15 y los 64 años, consumió drogas por lo menos en una ocasión en 2015. El cannabis es la droga más ampliamente usada, pero los opioides generaron gran impacto negativo en la salud. Los estimulantes de tipo anfetamínico se mantienen como la segunda droga más usada a nivel mundial, con un estimado de 35 millones de usuarios en el último año $(\underline{3}, 7)$.

El consumo de SPA en adolescentes puede estar condicionado por múltiples factores (5), algunos estudios han establecido que los principales factores asociados al consumo son: influencia del grupo de pares, disponibilidad de sustancias en el entorno, presencia de síntomas depresivos, abandono escolar, consumo en la familia, uso de sustancias "gatillo" como el tabaco y el alcohol y factores psicológicos y biológicos individuales ()ㅡㄴ, dichos aspectos contribuyen al deterioro de los lazos sociales y familiares y a la privación social (8) . Por lo que es un fenómeno complejo que no depende solamente de la persona que consume, es un proceso donde intervienen la familia, la sociedad y el entorno, y debe ser afrontado desde un enfoque integral. Para la comprensión de este fenómeno es necesario identificar y articular tres escenarios: la familia, la comunidad y la escuela, cuyas interacciones determinan la exposición al riesgo de consumo de sustancias psicoactivas ${ }^{4}$.

Este estudio es de vital importancia para identificar los factores asociados al consumo de sustancias psicoactivas y de ésta manera evidenciar la magnitud del problema, sus causas, sus efectos y a partir de esta información se puedan generar acciones conjuntas entre la institución educativa, los estudiantes, los padres de familia, las autoridades locales, regionales y departamentales, tendientes a la promoción y prevención del consumo de sustancias psicoactivas, de tal forma que contrarresten el flagelo de la drogadicción que ha ido en aumento y que causa daño a los jóvenes ${ }^{(9)}$, ya que existe un alto riesgo de enfermedades psiquiátricas entre las personas que abusan de las drogas, afectando significativamente no solo la calidad 
de vida de estas personas, sino que también crea dificultades especiales, particularmente en lo que respecta al tratamiento (8), hay que tener en cuenta que los adolescentes son más vulnerables a las adicciones y cualquier uso de sustancias psicoactivas en este grupo se considera un comportamiento de alto riesgo de tener efectos nocivos para la salud y aumenta la probabilidad de desarrollar abuso o dependencia a largo plazo $(10,11)$.

Es importante resaltar que por cada joven inmerso en el consumo de sustancias psicoactivas existirá un mayor número de adolescentes en riesgo, motivados por quienes ya están en el problema, esto se convierte en un círculo vicioso que aumenta el consumo de SPA en las poblaciones vulnerables, y que se ve fortalecido por las redes de microtráfico ${ }^{(12)}$, con graves implicaciones físicas, psicológicas, sociales, familiares, laborales, académicas, económicas y jurídicas ${ }^{(13)}$.

La Organización Mundial de la Salud (OMS) señala que cada año se producen 3,3 millones de muertes en el mundo, debido al uso nocivo del alcohol, que representan el 5,9\% de todas las defunciones. Por otro lado, 6 millones de personas murieron a causa del tabaco y alrededor de 200 mil murieron a causa del uso de otras drogas Las consecuencias del uso y del abuso de drogas no solo se limitan a esas muertes particulares, su efecto se propaga como una ola (14).

Los estudios del Observatorio de Drogas de Colombia (ODC) muestran que el consumo de drogas se está incrementando en el país, no solo porque las personas las consumen, sino porque el mercado es cada vez más amplio y diverso (그. En Colombia anualmente se presenta un incremento en la prevalencia del uso de marihuana a nivel nacional desde un $2.1 \%$ el 2008 , a $3.3 \%$ en el 2013. Este incremento significativo se observa tanto en hombres (3.5\% a $5.4 \%$ ) como en mujeres (de $0.8 \%$ a $1.3 \%$ ), en los grupos de edad de 12 a 17 años (2.7\% a $4.3 \%$ ) y de 18 a 24 años (de un 5\% el 2008 a un 8.2\% el 2013), y en los estratos 1, 2 y 3 . En el 2013 se estableció que $11.5 \%$ de la población colombiana usó marihuana alguna vez en la vida teniendo como precedente que en el año 2008 la cifra se mantuvo alrededor de $7.9 \%$, señalando de esta manera un aumento de casi cuatro puntos $(\underline{15,16)}$.

De acuerdo con el Reporte de Drogas de Colombia del 2015, las sustancias ilícitas más consumidas en Colombia son en su orden: la marihuana, la cocaína, el basuco y el éxtasis. Según el mismo estudio, se evidencia un aumento en el consumo de heroína en los últimos años, determinado por el incremento en la demanda de tratamiento por consumo en siete territorios: Santander de Quilichao, Armenia, Medellín, Pereira, Cúcuta, Cali y Bogotá (2).

El objetivo de esta investigación fue determinar los factores asociados al uso de sustancias psicoactivas en pacientes atendidos por el servicio de psiquiatría en el Hospital San Rafael durante el año 2018.

\section{MÉTODOS}

2.1. Diseño del estudio: Estudio analítico de casos y controles hospitalarios. 
2.2. Universo, muestra y protocolo de muestreo: El universo del estudio fueron todos los pacientes atendidos en el servicio de Psiquiatría del Hospital San Rafael durante el año 2018, con el fin de establecer grupos comparables.

El cálculo de tamaño de muestra se realizó mediante el programa Epi-info versión 7.2, estableciendo una potencia del 85\%, frecuencia de exposición en caso del 56\%, frecuencia de exposición en controles de 38\%, intervalo de confianza 95\%, Odds ratio: 2.0 y una relación de casos - controles 1:2. Teniendo en cuenta esta información se revisaron un total de 309 historias clínicas, divididas en 103 casos y 206 controles. Estos fueron seleccionados mediante un muestreo probabilístico de tipo aleatorio simple que se realizó en el programa Excel versión 2013. Los casos y controles fueron pareados según la edad, puesto que se seleccionó primero al grupo casos y, posteriormente se estableció que por cada caso se iban a tomar dos personas controles con la misma edad.

2.3. Criterios de selección de los grupos: El universo poblacional fueron personas entre 12 a 65 años de edad, atendidos en el servicio de Psiquiatría del Hospital San Rafael entre el 01 de enero a 31 de diciembre del 2018. Los casos fueron idenficados mediante los Códigos internacionales de Enfermedades ( $\mathrm{CIE}-10)$ relacionado con uso, abuso 0 intoxicación por sustancias psicoactivas que estaban entre el código F10.0 hasta el F19.9, mientras que los controles pertenecían a las otras patologías diagnosticadas y tratadas por el servicio de psiquiatría. Finalmente se excluyeron aquellos pacientes con historia clínica incompleta, los que tenían diagnóstico de alguna patología psiquiátrica y a la vez habían consumido alguna sustancias psicoactiva.

2.4. Variables: La variable dependiente era el consumo de sustancias psicoactivas sin tener presente el tipo de sustancia consumida, mientras que las variables independientes fueron la edad, genero, área de residencia, tener hijos, factores psicosociales como estar empleado, antecedente de abuso sexual, antecedente de abuso físico, violencia intrafamiliar, consumo de sustancia psicoactiva por un familiar, maltrato físico o psicológico en la actualidad, abandono de la familia, núcleo familiar disfuncional por apgar familiar y peleas entre los padres.

2.5. Análisis estadístico. La base de datos fue registrada en Excel versión 2013 y se analizó en el paquete estadístico SPSS versión 22.0. El análisis univariado se realizó mediante una descripción a cada grupo (caso y control), donde se determinaron frecuencias absolutas y relativas en las variables categóricas, mientras que se calcularon medidas de tendencia central (media, mediana) y medidas de dispersión (desviación estándar y rango intercuartil) en las variables cuantitativas. El análisis bivariado se realizó mediante tablas tetracoricas, con medición de Chi cuadrado de Pearson y Odds Ratio con su respectivos IC al 95\%, finalmente, en el análisis multivariado se realizó un modelo de regresión logística binaria, donde se añadieron al modelo los factores estudiados que mostraron asociación en el análisis bivariado del presente estudio y aquellos que tengan un valor representativo teórico.

2.6. Sesgos: Los sesgos en el presente estudio fueron: 1). Sesgo de mala clasificación, es decir, en el que un caso puede ser escogido como control o viceversa. Para evitar este tipo de sesgo, se establecieron criterios de inclusión y exclusión claros para su diferenciación. 
2). Sesgo de medición, en el cual los investigadores hacen más detallada la búsqueda de información en alguno de los dos grupos; para corregirlo se utilizó una ficha de recolección que fue aplicada por todos los investigadores al momento de recolectar la información. 3). Sesgo de selección, que se produce cuando la inclusión de los casos o los controles depende, en cierta medida, de la exposición de interés. Para evitar esto se seleccionaron los controles por medio de un muestreo probabilístico aleatorio simple. 4). Sesgo de confusión, donde se establece que dos variables pueden generar riesgo en el evento analizado tanto en el grupo caso como en el grupo control, por este motivo se decidió analizar los datos por medio de un método de regresión logística binaria. Adicionalmente y teniendo en cuenta que la población control eran pacientes atendidos por el servicio de psiquiatría se excluyeron aquellos pacientes con patología psiquiátrica que consumieron sustancias psicoactivas. Otro elemento relevante, es que existe una posible asociación entre los factores psicosociales que favorecen el consumo de sustancias y la aparición de patologías psiquiátricas, por ende, estas fueron las variables de confusión que requirieron controlarse durante los análisis de datos. 5). Sesgo de vacíos en el registro de la información, en el que los datos son obtenidos de los registros de las historias clínicas con la posibilidad de que la información recolectada esté incompleta, por este motivo se eliminaron aquellos pacientes con datos incompletos.

2.7. Aspectos éticos: Basados en la resolución 8430 de 1993, donde se establecen las normas en salud, se considera un estudio sin riesgo, puesto que se basa en la revisión del historial clínico de los pacientes. (17) Adicionalmente, se solicitó autorización al comité de ética e investigación del Hospital San Rafael con acta de aprobación №2-2019-05, al ser la entidad encargada de la custodia y manejo de la información de los pacientes, desde el que no se obtuvo objeciones con respecto a los datos de menores de edad, por tratarse de un estudio retrospectivo y en el que se va a cuidar la custodia de la información.

\section{RESULTADOS}

\section{Selección de participantes}

921 personas fueron atendidas por el servicio de psiquiatría del Hospital San Rafael de Tunja entre el 1 de enero y el 31 de diciembre del 2018 según la base de datos de esta institución, de los cuales 691 cumplían el criterio de edad y tenían entre los 12 y 65 años de edad. De estos se identificaron un total de 187 casos por medio de los códigos CIE 10 y 504 posibles controles.

De esta forma y contando con la lista se realizó un muestreo probabilístico aleatorio simple para la selección de los participantes (casos y controles), donde se obtuvo un total de 103 casos y 206 controles. Sin embargo, durante el proceso de revisión de historias se eliminaron 8 casos ( 3 no contaban con todas la variables incluidas en el estudio y 5 eran pacientes que habían presentado esquizofrenia posiblemente secundario al uso de sustancias psicoactivas) y 82 controles (8 con historias clínicas incompletas, 14 pacientes habían consumido sustancias psicoactivas y 60 pacientes no concordaba con las edades del grupo caso para el respectivo pareamiento); razón por la cual se realizó una nueva selección aleatoria para la elección de los participantes faltantes ( 8 casos y 82 controles), de este modo se alcanzó el tamaño de la muestra sin presentar pérdidas. 


\subsection{Análisis univariado}

El promedio de edad de la población fue de 29.52 años DE \pm 15.05 en ambos grupos. La edad de inicio de consumo de sustancias psicoactivas promediaba a los 17.8 años $\mathrm{DE} \pm 5.2$ años. El tiempo que llevaban consumiendo fue en promedio de 12.05 años $\mathrm{DE} \pm 9.87$ años. En la tabla 1 se muestran las variables cualitativas de ambos grupos.

Tabla 1. Análisis univariado en las variables cualitativas.

\begin{tabular}{|c|c|c|}
\hline Variable & $\begin{array}{c}\text { Grupo caso } \\
(n=103)\end{array}$ & Grupo control $(n=206)$ \\
\hline Género femenino & $17.4 \%$ (18 casos) & $55.8 \%$ (115 controles) \\
\hline Residencia urbana & $78.6 \%$ (81 casos) & $51.9 \%$ (107 controles) \\
\hline Tiene hijos & $24.3 \%$ (25 casos) & $43.7 \%$ (90 controles) \\
\hline Desempleado & $58.2 \%$ (90 casos) & $69.9 \%$ (144 controles) \\
\hline $\begin{array}{c}\text { Antecedente de abuso } \\
\text { sexual }\end{array}$ & $0.9 \%$ ( 1 casos $)$ & 1.5\% (3 controles) \\
\hline $\begin{array}{l}\text { Antecedente de abuso } \\
\text { físico }\end{array}$ & $21.4 \%$ (22 casos) & $12.1 \%$ (25 controles) \\
\hline Violencia intrafamiliar & $38.8 \%$ (40 casos) & $21.4 \%$ (44 controles) \\
\hline $\begin{array}{c}\text { Consumo de SPA por } \\
\text { un familiar }\end{array}$ & $41.7 \%$ (43 casos) & $17 \%$ (35 controles) \\
\hline Maltrato físico actual & $23.3 \%$ (24 casos) & $8.7 \%$ (18 controles) \\
\hline $\begin{array}{c}\text { Maltrato psicológico } \\
\text { actual }\end{array}$ & $36.9 \%$ (38 casos) & $14.6 \%$ (30 controles) \\
\hline $\begin{array}{c}\text { Negligencia o } \\
\text { abandono familiar }\end{array}$ & $40.8 \%$ (42 casos) & $23.8 \%$ (49 controles) \\
\hline $\begin{array}{l}\text { Núcleo familiar } \\
\text { disfuncional }\end{array}$ & $69 \%$ (71 casos) & $47.5 \%$ (98 controles) \\
\hline Peleas entre los padres & $41.7 \%$ (43 casos) & $12.1 \%$ (25 controles) \\
\hline
\end{tabular}

Fuente: Propia de los autores.

\subsection{Análisis bivariado}

En la tabla 2 se presentan los OR crudo y su intervalo de confianza al 95\%. Se encontró asociación con algunos factores de riesgo como la pelea entre padres, consumo de sustancias por parte de un familiar, vivir en zona urbana, sufrir maltrato psicológico y físico, estar desempleado, núcleo familiar disfuncional, violencia intrafamiliar y abandono de la familia. Se detectaron algunos posibles factores protectores como el sexo femenino y tener hijos.

\subsection{Análisis multivariado}

En el análisis multivariado, controlando los factores de confusión (factores psicosociales) se encontró que la pelea entre padres frente al consumidor, consumo de sustancias por parte de un familiar, vivir en zona urbana, estar desempleado, núcleo familiar disfuncional son factores de riesgo, mientras que ser del sexo femenino y tener hijos son factores que disminuyen la probabilidad de consumir sustancias psicoactivas. 
Tabla 2. Análisis bivariado de los factores asociados al consumo de sustancias psicoactivas.

\begin{tabular}{|c|c|c|}
\hline Variable & Odds ratio & IC 95\% \\
\hline Peleas entre los padres & 5.18 & $2.92-9.20$ \\
\hline Consumo de SPA por un familiar & 3.50 & $2.05-5.97$ \\
\hline Residencia urbana & 3.93 & $1.98-5.94$ \\
\hline Maltrato psicológico & 3.37 & $1.93-5.88$ \\
\hline Maltrato físico & 3.17 & $1.63-6.17$ \\
\hline Desempleado & 2.98 & $1.57-5.90$ \\
\hline Núcleo familiar disfuncional & 2.44 & $1.48-4.02$ \\
\hline Violencia intrafamiliar & 2.33 & $1.39-3.92$ \\
\hline Abandono de la familia & 2.20 & $1.32-3.66$ \\
\hline Antecedente de abuso físico & 1.96 & $1.47-3.69$ \\
\hline Antecedente de abuso sexual & 0.66 & $0.06-6.45$ \\
\hline Tiene hijos & 0.41 & $0.24-0.70$ \\
\hline Género femenino & 0.16 & $0.094-0.21$ \\
\hline
\end{tabular}

Fuente: Propia de los autores.

Tabla 3. Análisis multivariado con regresión logística binaria.

\begin{tabular}{|c|c|c|}
\hline Variable & $\begin{array}{c}\text { Odds ratio } \\
\text { ajustado }\end{array}$ & IC 95\% \\
\hline Peleas entre los padres & 7.25 & $1.16-25.48$ \\
\hline Consumo de SPA por un familiar & 2.81 & $1.07-5.75$ \\
\hline Residencia urbana & 7.52 & $1.62-22.63$ \\
\hline Maltrato psicológico actual & 6.72 & $0.95-12.73$ \\
\hline Maltrato físico actual & 4.86 & $1.00-8.92$ \\
\hline Desempleado & 1.65 & $1.18-9.52$ \\
\hline Núcleo familiar disfuncional & 3.01 & $1.35-7.65$ \\
\hline Violencia intrafamiliar & 1.52 & $0.56-2.75$ \\
\hline Negligencia o abandono familiar & 3.72 & $0.98-7.92$ \\
\hline Antecedente de abuso físico & 4.50 & $0.75-5.8$ \\
\hline Tiene hijos & 0.67 & $0.15-0.89$ \\
\hline Género Femenino & 0.55 & $0.004-0.87$ \\
\hline
\end{tabular}

Fuente: Propia de los autores.

\section{DISCUSIÓN}

Los participantes que habían consumido sustancias psicoactivas pertenecían en la mayoría de los casos al sexo masculino (82.5\%), hecho que concuerda con los datos reportados por Sierra y colaboradores $(78.4 \%) \stackrel{(18)}{ }$, Martinez y cols $(79.2 \%) \stackrel{(19)}{\text { y, Medina y cols. }(81.2 \%)} \stackrel{(20)}{ }$. La edad 
promedio de inicio de consumo se relaciona con varios estudios donde indican que en su mayoría son menores de edad $(\underline{21}, \underline{22})$.

Con esta investigación se pudo determinar que los factores familiares, la relación en la familia, la convivencia con los padres y el tiempo que se les dedique a los hijos tienen un gran peso e influye en el inicio de consumo de drogas ilegales $(\underline{23}, \underline{24}, \underline{25}, 26)$.

Las peleas o conflictos familiares frente a los menores y el hecho de convivir en un núcleo familiar disfuncional son factores de riesgo, y son datos que se han reportado en otras investigaciones ${ }^{(6,27)}$. Se he evidenciado que la separación de los padres biológicos no es un factor que predisponga al consumo de SPA, sin embargo, un ambiente hostil e inestable, relacionado con la violencia y la baja estabilidad económica si afecta considerablemente y facilita la exposición de los menores a las drogas ilícitas (28).

Adicionalmente, se encontró que el consumo de sustancias por parte de un familiar representa un incremento significativo del riesgo de consumo de sustancias psicoactivas. Estos datos están acordes con lo reportado por otros investigadores $(\underline{29}, \underline{30}, \underline{31}, \underline{32})$, lo cual se puede ver reflejado en el aprendizaje por imitación; para Albert Bandura este tipo de aprendizaje se da por la exposición a acciones y modelos que se asimilan como propios $(\underline{33})$.

Las personas que viven en zonas urbanas, tiene mayor riesgo de consumo de sustancias ilícitas (7.52), esto puede estar asociado con la exposición y la facilidad que tienen las personas para adquirir las drogas en esta zona, puesto que las áreas de expendios se encuentran principalmente dentro de las grandes ciudades, además que se da en un contexto donde un amigo o alguien cercano puede incitar o facilitar la "curiosidad" y la adquisición de las drogas ilegales $(\underline{34}, \underline{35})$.

Uno de los factores que afecta a la población con conductas de dependencia a SPA es el desempleo, puesto que potencian la exclusión y dificulta la posibilidad de reinsertarse en la vida laboral, además de aumentar la pérdida de competitividad, precariedad y temporalidad en el trabajo, mayores niveles de marginalidad, cambio en los modelos familiares y debilidad en las redes de apoyo que tiene la persona $(\underline{36})$.

Dentro de los factores analizados, se encontró que el sexo femenino es un factor protector $(0.55)$, dato que concuerda con lo reportado por Saravia $(0,62)$. Indicando que aún se mantiene el modelo tradicional de roles femenino y masculino $(\underline{37})$.

Finalmente, se encontró que las personas con hijos tienen un menor riesgo de exponerse al consumo de drogas de tipo ilegal, sin embargo, no hay estudios que reporten este antecedente, sin embargo, se puede considerar que se asocia a una mayor responsabilidad por parte del progenitor.

El consumo de sustancias psicoactivas afecta los distintos entornos del desarrollo de la persona, tales como la salud, la economía, la funcionalidad en la familia o el hogar, siendo este último uno de los factores que más influye para esta patología, por lo que se destaca como un aspecto fundamental. Es por esto que se debe fortalecer a la persona desde las relaciones en el hogar, adicionalmente se debe tener en cuenta que la vivienda y el ambiente que rodea a la 
persona puede influir, por lo que la residencia cerca a zonas de expendios de drogas y tener amigos o familiares que consuman incrementa este riesgo $(\underline{38}, \underline{39}, \underline{40})$.

Se cumplió el objetivo de la investigación, donde se determinó que los factores de riesgo asociados al consumo de sustancias psicoactivas en orden de importancia fueron los conflictos familiares frente al menor de edad, antecedente de consumo de sustancias por parte de un familiar, vivir en zona urbana, y núcleo familiar disfuncional, mientras que el sexo femenino y tener hijos son factores que disminuyen la probabilidad de consumir sustancias psicoactivas. La validez interna es adecuada para la población que sea atendida por el servicio de psiquiatría del Hospital San Rafael de Tunja, mientras que para la validez externa y la generalización a otros servicios se debe tener en cuenta que cumpla con los criterios analizados en el estudio. Es de vital importancia aclarar que, a pesar de los intentos por controlar los sesgos, el tipo de diseño investigativo, la recolección retrospectiva y la falta de información pudo alterar los resultados.

\section{CONCLUSIONES}

Los factores de riesgo para el consumo de sustancias psicoactivas son los conflictos familiares, antecedente de consumo de sustancias por parte de un familiar, vivir en zona urbana y núcleo familiar disfuncional; mientras que el sexo femenino y tener hijos son factores que disminuyen la probabilidad de consumir sustancias psicoactivas. Basado en estos resultados se debe plantear una adecuación en la atención del paciente en atención primaria con el fin de detectar estos factores y controlarlos de manera temprana, siendo un paso importante para la comunidad en general y que fortalece las políticas de salud pública.

Desde la consulta tanto en los pacientes que han consumido como en los que no lo han hecho, es importante tener presente los factores psicosociales que puedan influir en la aparición de esta entidad patológica con el fin de hacer una detección temprana y evitar que se llegue al consumo de SPA. Los profesionales de la salud siendo primera línea para atención en salud deben tener presente la prevención como primera intervemción.

Contribución de los autores/Author Contributions: JCAM, LJVR, PAB, SAGL, MAFL, GVAC, AFBS: Conceptualización, metodología, software, validación, análisis formal, investigación, recursos, curación de datos, escritura: preparación del borrador original, escritura: revisión y edición, supervisión. Todos los autores han leído y aceptado la versión publicada del manuscrito.

Fondos: Esta investigación no recibió fondos externos

Conflictos de intereses: Los autores declaran no tener ningún conflicto de intereses.

\section{REFERENCIAS}

1. Castaño-Castrillón JJ, García S, Luna J, Morán M, Ocampo D, Ortiz L. Estudio de factores asociados y prevalencia de consumo de sustancias psicoactivas ilegales en estudiantes de una universidad colombiana. Rev Fac Med Univ Nac Colomb. 2017;65(1):23-30 
2. Perea BME, Pérez EAC, Arango DAG, Villa CFH. Liderazgo y sistemas de información: análisis aplicado al seguimiento del consumo de sustancias psicoactivas en Colombia [Internet]. 2021; 43: 327-37. Available from: https://www.proquest.com/openview/bd150cfbf7ccfb6ea343815395c5b52e/1?pqorigsite $=$ gscholar \&cbl $=1006393$

3. Córdoba-Paz EG, Betancourth-Zambrano S, Tacán-Bastidas LE. Consumo de sustancias psicoactivas en una universidad privada de Pasto, Colombia/ Psychoactive substances at a private university from Pasto, Colombia. PSICOGENTE. 2017; 20(38):308-19.

4. Álvarez-López ÁM, Carmona-Valencia NJ, Pérez-Rendón ÁL, Jaramillo-Roa A. Factores psicosociales asociados al consumo de sustancias psicoactivas en adolescentes de Pereira, Colombia. Univ salud. 2020; 22(3):213-22.

5. Ferrel Ortega FR, Ferrel Ballestas L, Alarcón Baquero A, Delgado Arrieta K. El consumo de sustancias psicoactivas como indicador de deterioro de la salud mental en jóvenes escolarizados. Psychol. 2016; 10(2):43-54.

6. Espinosa Soto K, Hernández Carrillo M, Cassiani CA, Cubides Munevar ÁM, Martínez Cardona $\mathrm{M}$ del $\mathrm{C}$. Factors related with psychoactive substance use in an educational institution in Jamundí Valle, Colombia. Rev Colomb Psiquiatr. 2016; 45(1):2-7.

7. Ministerio de Justicia y del Derecho y el Observatorio de Drogas de Colombia (ODC). Reporte de drogas de Colombia [Internet]. Bogotá (Colombia): Minjusticia; 2017 [cited 2020 Mar 13]. Available from: http://www.odc.gov.co/Portals/1/publicaciones/pdf/odclibroblanco/reporte_drogas_colombia_2017.pdf

8. Fernandes MA, Feitosa CDA, Mendes PN, Oliveira ALCB de, Silva JS e. Hospitalizations due to psychoactive substances usage: a psychiatric hospital study. Rev Pesqui Cuid é Fundam Online. 2020; 12:1132-8.

9. Aguirre-Guiza NC, Aldana-Pinzón OB, Bonilla-lbáñez CP. Factores familiares de riesgo de consumo de sustancias psicoactivas en estudiantes de una institución de educación media técnica de Colombia. Rev Salud Pública (Bogotá). 2017; 19(1):3-9.

10. Vilugrón Aravena F, Hidalgo-Rasmussen CA, Molina G T, Gras Pérez ME, Font-Mayolas $\mathrm{S}$. Psychoactive substances use and health-related quality of life among school age adolescents. Rev Med Chil. 2017; 145(12):1525-34.

11. Espinosa K, Hernández M, Cassiani A, Cubides A, Martínez M. Factores relacionados con el consumo de sustancias psicoactivas en una institución educativa de Jamundí Valle, Colombia. Rev Colomb Psiquiat. 2016; 45(1):2-7.

12. Mousavi F, Garcia D, Jimmefors A, Archer T, Ewalds-Kvist B. Swedish high-school pupils' attitudes towards drugs in relation to drug usage, impulsiveness and other risk factors. Peer J. 2014; 2:e410-415.

13. Wongtongkam N, Ward PR, Day A, Winefield A. The influence of protective and risk factors in individual, peer and school domains on Thai adolescents' alcohol and illicit drug use: A survey. Addict Behav. 2014; 39:1447-51.

14. Olive YC, Gómez OM, Lio-Coo VT. Ansiedad y depresión en pacientes adictos a sustancias psicoactivas Anxiety and depression in patients addicted to psychoactive substances. Available from: http://scielo.sld.cu/pdf/mil/v49n1/1561-3046-mil-49-01e492.pdf

15. Unodc.org [Internet]: Col. Estudio Nacional de Consumo de Sustancias Psicoactivas; 2013.

[Citado

2019Mayo

04].

Disponible 
en:mailto:https://www.unodc.org/documents/colombia/2014/Julio/Estudio de Consum o UNODC.pdf

16. Valencia Álzate DC, Mancilla Jiménez JC. Consumo de sustancias psicoactivas en estudiantes de 10 y $11^{\circ}$ de una institución educativa del corregimiento de robles, municipio de Jamundí, valle del cauca. 2020. Universidad Santiago de Cali; 2020.

17. La cual se establecen las normas científicas $P$, la investigación en salud. T y. AP. RESOLUCION NUMERO 8430 DE 1993 [Internet]. Gov.co. [cited 2021 Sep 21]. Available from: https://www.minsalud.gov.co/sites/rid/Lists/BibliotecaDigital/RIDE/DE/DIJ/RESOLUCIO N-8430-DE-1993.PDF

18. Sierra D, Pérez M, Pérez A. Representaciones sociales en jóvenes consumidores y no consumidores de sustancias psicoactivas. Rev. Adicciones, Sección Latinoamericana. 2005; 17:349-60

19. Martínez J, Amaya W, Campillo H. Consumo de sustancias psicoactivas en adolescentes, Bucaramanga, Colombia 1996-2004. Rev. Salud Pública. 2007;9:215-29.

20. Medina O, Rubio L. Consumo de sustancias psicoactivas (SPA) en adolescentes farmacodependientes de una fundación de rehabilitación colombiana. Estudio descriptivo. Rev. Colomb. Psiquiat. 2012; 41(3): 550-561.

21. Mousavi F, Garcia D, Jimmefors A, Archer T, Ewalds-Kvist B. Swedish high-school pupils' attitudes towards drugs in relation to drug usage, impulsiveness and other risk factors. Peer J. 2014; 2:e410.

22. Martínez M, Martínez J, López M, Attas D. Estudio sobre el consumo de drogas en estudiantes de la provincia de Valencia, Espana. Vertex (B Aires). 2013; 24:333-41.

23. Oral M, Rodríguez F, Ovejero A. Correlatos psicosociales del consumo de sustancias psicoactivas en adolescentes españoles. Rev. Salud pública de México. 2010; 52:406415.

24. Mosqueda A, Carvalho M. Factores protectores y de riesgo familiar relacionados al fenómeno de drogas, presentes en familias de adolescentes tempranos de valparaíso Chile. Rev latino-am enfermagem. 2011; 19: 789-795.

25. Becoña E, Fernandez A. ¿Cómo influye la desorganización familiar en el consumo de drogas de los hijos?. Rev. Adicciones. 2012; 24(3):253-268.

26. Riofrío R, Castanheira I. Consumo de drogas en los jóvenes de la ciudad de Guayaquil, Ecuador. Rev. Latino-am enfermagem. 2010; 18:598-605.

27. Tuesca R, Borda M. Violencia física marital en Barranquilla (Colombia): prevalencia y factores de riesgo. Gac Sanit. 2003; 17:302-308.

28. Cooley M, Quille T, Griffin R, Stuart E, Bradshaw C, Furr D. Efectos de la exposición de los adolescentes a la violencia en la comunidad: el proyecto more. Psychosocial intervention. 2011; 20(2): 131-148.

29. Brook J, Balka E, Crossman A, Dermatis H, Galanter M, Brook D. The relationship between parental alcohol use, early and late adolescent alcohol use, and young adult psychological symptoms: a longitudinal study. Am j Addict.2010; 19 (6): 534-542.

30. Caicedo R, Moreno D, Ferreira J, Mendoza L, Rueda L, Murillo P. Diagnóstico para el consumo de sustancias psicoactivas en adolescentes. Rev Cienc cuidado. 2012; 9(2):26-35.

31. Joo S, Johnson B. The Effects of childhood exposure to drug users and religion on drug use in adolescence and young adulthood. Youth society.2011; 43(4): 1220-1245.

32. Díaz K. medición y caracterización de factores familiares, individuales y ambientales en 
consumo y no consumo de drogas en adolescentes. [tesis de doctorado]. Universidad Nacional de Colombia, facultad de enfermería, Bogotá. 2011.

33. Rodríguez, F.Estrategias metodológicas para la enseñanza de educación vial: fundamentada en el aprendizaje vicario de Albert Bandura. Repositorio UTMACH. 2017; 117.

34. Cid P, Pedrao L. Factores familiares protectores y de riesgo relacionados al consumo de drogas en adolescentes. Rev Latino-am Enfermagem. 2011; 19:738-745.

35. Pons J.El modelado familiar y el papel educativo de los padres en la etiología del consumo de alcohol en adolescentes. Rev Esp Salud Pub. 1998; 72(3):250-259.

36. Becoña, E. Bases científicas de la prevención de las drogodependencias, Plan Nacional sobre Drogas, 2002; 377.

37. Da Silva M, Rumbao F,Benitez G, García R, Rodríguez T. Consumo de alcohol y relaciones sexuales en adolescentes del sexo femenino. Rev Facultad de Medicina. 2001; 24:135-139.

38. Acosta L, Fernández A, Pillon S. Factores sociales para el uso de alcohol en adolescentes y jóvenes. Rev Latinoamericana Enferagem. 2011; 19:771-781.

39. Saiz J. Estudio empírico de las variables de la teoría de la conducta plantificada como factores de riesgo para el consumo de cocaína en tres grupos diferentes. Rev Adicciones. 2009; 21:187-194.

40. Becoña E, Fernandez A. ¿Cómo influye la desorganización familiar en el consumo de drogas de los hijos? Rev. Adicciones. 2012; 24(3):253-268. 